\section{A rare cause of pericardial effusion and ascites: POEMS syndrome}

\author{
Bilal Katipoglu, ${ }^{1}$ Zeynep Katipoğlu, \\ Ihsan Ates, ${ }^{1}$ Gokhan Yirgin, ${ }^{1}$ \\ Burak Furkan Demir, ${ }^{1}$ Fatih Acehan ${ }^{1}$ \\ ${ }^{1}$ Department of Internal Medicine, \\ Ankara Numune Training and Research \\ Hospital, Ankara; ${ }^{2}$ Department of \\ Ophthalmology, Necmettin Erbakan \\ University, Konya, Turkey
}

\begin{abstract}
POEMS syndrome is an important paraneoplastic syndrome associated with multisystem involvement. Extravascular volume overload like pericardial effusion and ascites has a broad differential diagnosis. In addition, it may be initial presentation of disease. For that reason, this case report is highlight to warn of different forms of presentation of poems syndrome.
\end{abstract}

\section{Introduction}

POEMS syndrome is a rare paraneoplastic syndrome due to underlying plasma cell dyscrasia. It is characterized by polyneuropathy, organomegaly, endocrinopathy, monoclonal protein and skin changes. It is initially presented with extravascular volume overload. ${ }^{1}$ We report a rare case of pericardial effusion and refractory ascites appearing as the initial manifestations of POEMS syndrome.

\section{Case Report}

A 48-year-old female was admitted to a hospital with dyspnea and abdominal discomfort. Her medical history included diabetes mellitus and hypothyroidism for two years. She also complained of lower limb numbness and weakness. Moreover, her body weight decreased $25 \mathrm{~kg}$ in six months.

Physical examination showed that she had cervical lympadenopathy, abdominal ascites, splenomegaly, peripheral edema, hyperpigmented skin, white nails and clubbing. Neurological examination was notable a symmetrical lower limb weakness and vibratory and proprioceptive loss.

Hematological tests showed hemoglobin $10.2 \mathrm{~g} / \mathrm{dL}$ (12-16), white cell count 9000 $\mu \mathrm{L}$ and platelet counts were in normal range. Endocrine tests confirmed hypothyroidism and diabetes mellitus. Other biochemical and hormonal test was normal. $24 \mathrm{~h}$ urine total protein was $0.84 \mathrm{~g}$. Serum autoimmune antibodies (DSDNA, ENA, ANA, ACL, MPO-ANCA and PR3-ANCA) and tumor markers (AFP, CEA, CA19-9, and CA125) were negative. Autoimmune markers and viral panel were negative. Serum-ascites albumin gradient (SAAG) is $12 \mathrm{~g} / \mathrm{L}$. Biochemical and cytological analysis of ascitic fluid showed no evidence of infection and malignancy. CT scan identified hepatomegaly, splenomegaly, lymphadenopathy and ascites. Endoscopy revealed portal hypertension gastropathy and normal colon. Echocardiography revealed pulmonary artery pressure of $45 \mathrm{~mm} / \mathrm{hg}$, ejection fraction $72 \%$ and pericardial effusion $1.2 \mathrm{~cm}$. Monoclonal immunoglobulin a lambda protein was detected in the serum by protein electrophoresis and immunofixation. Bone marrow aspirate and biopsy showed plasmocytosis (5\%) with abnormal morphology. Biopsy of her cervical lymph nodes indicated chronic lymphadenitis. Electromyoraphic studies showed symmetric sensorimotor neuropathy involving the lower extremities. This case was also consulted with the department of ophthalmology physicians who detected mild bilateral optic disc swelling. Serum vascular endothelial growth factor (VEGF) levels were significantly raised at $3769 \mathrm{pg} / \mathrm{mL}$ (ref: <771).

After diagnosed as POEMS syndrome she was commenced on a chemotherapy regime of cyclophosphamide, thalidomide and dexamethasone with aim of autologous peripheral stem cell transplantation. She made good clinical response and there was significant improvement after treatment.

\section{Discussion}

POEMS syndrome is a rare clonal plasma cell disease. ${ }^{1}$ The mechanism of POEMS syndrome is not fully understood, although pro-inflammatory and VEGF appears to be a important role in pathogenesis. $^{2}$

It is demonstrated that platelets or plasma cells were major sources of VEGF which induces endothelial cells proliferation, and microvascular hyperpermeability and play an important role in angiogenesis. ${ }^{3}$ Like in our cases, VEGF level was significantly elevated in the early stages. Perhaps microvascular hyperpermeability contributes to extravascular volume overload. This can be presented like pleural effusion,
Correspondence: Bilal Katipoglu, Ankara Numune Training and Research Hospital, Department of Internal Medicine, Sihhiye, 06100, Ankara, Turkey.

Tel.: +90.312.5084552 - Fax: +90.312.3113958. E-mail: drbila107@gmail.com

Key words: ascites, pericardial effusion, poems syndrome.

Contributions: the authors contributed equally.

Conflict of interest: the authors declare no potential conflict of interest.

Received for publication: 27 August 2017. Accepted for publication: 3 November 2017.

This work is licensed under a Creative Commons Attribution-NonCommercial 4.0 International License (CC BY-NC 4.0).

(C) Copyright B. Katipoglu et al., 2017

Licensee PAGEPress, Italy

Hematology Reports 2017; 9:7384

doi:10.4081/hr.2017.7384

pericardial effusion and ascites. Moreover, VEGF level decrease significantly after successful treatment. ${ }^{2}$ Other pro-inflammatory cytokines like Interleukin (IL)-1 $\beta$, IL6 , Tumor Necrosis Factor (TNF)- $\alpha$ have also been comprised in POEMS syndrome disease activity implicated. ${ }^{4}$

Ascites is a common complication of POEMS syndrome and has characteristics of non-portal hypertension, based on low SAAG. In our case report, SAAG of ascites fluid is over $12 \mathrm{~g} / \mathrm{L}$, accompanied by splenomegaly and portal hypertension gastropathy. All evidence confirmed the presence of portal hypertension. Portal hypertension is uncommon in POEMS syndrome. ${ }^{5}$ As it is highlighted by our case, portal hypertension was probably associated with POEMS syndrome. No signs suggested the presence of an other causes of portal hypertension. Although the mechanism portal hypertension remains unknown, the association may be not occasional.

\section{Conclusions}

Diverse clinical manifestations of POEMS syndrome make it a delayed diagnosis. This case report emphasized that, in differential diagnosis of pericardial effusion and ascites, POEMS syndrome should be considered especially when other symptoms such as organomegaly, numbness, endocrine alteration, and skin changes also present. 


\section{References}

1. Dispenzieri A. Poems syndrome: 2017 update on diagnosis, risk stratification, and management. Am J Hematol 2017;92:814-29.

2. Scarlato M, Previtali SC, Carpo M, et al. Polyneuropathy in poems syndrome: role of angiogenic factors in the pathogenesis. Brain 2005; 128:1911.

3. Endo I, Mitsui T, Nishino M, et al. Diurnal fluctuation of edema synchronized with plasma VEGF concentration in a patient with poems syndrome. Intern Med 2002;41:1196.

4. Tomas JF, Giraldo P, Lecumberri R, Nistal S. Poems syndrome with severe neurological damage clinically recovered with lenalidomide. Haematologica 2012;97:320-2.

5. Cui RT, Yu SY, Huang XS, et al. The characteristics of ascites in patients with poems syndrome.

Ann 